\title{
Revisiting urea-based gelators: strong solvent- and casting-microstructure dependencies and organogel processing using an alumina template $\dagger$
}

Received (in Montpellier, France) 28th April 2014,

Accepted 19th June 2014

DOI: 10.1039/c4nj00681j

www.rsc.org/njc

\author{
Thanh-Loan Lai, ${ }^{a}$ David Canevet, ${ }^{* a}$ Yasser Almohamed, ${ }^{a}$ Jean-Yves Mévellec, ${ }^{b}$ \\ Régis Barillé, ${ }^{a}$ Narcis Avarvarii ${ }^{* a}$ and Marc Sallé ${ }^{* a}$
}

\begin{abstract}
Urea-based gelators have been thoroughly characterized through various techniques and exhibit a strong solvent-structuration dependency in both the gel and the xerogel states. In a ground-breaking manner, gels were introduced in alumina membranes, which act as templates, in order to shape these materials and force the alignment of the corresponding self-assembled nanofibers by confinement.
\end{abstract}

\section{Introduction}

Self-assembled nanofibers are monodimensional objects made up of molecules that tend to form well-organized materials at the mesoscopic scale. ${ }^{1}$ Such compounds display great potential since they may find applications in fields as different as optoelectronics or pharmaceutics. Indeed, their large aspect-ratio and their crystalline character make them nice candidates as miniaturized electrical wires provided a relevant functionalization. ${ }^{2}$ Additionally, gels, which result from the self-assembly of nanofibers within a solvent, are from now on recognized as relevant media to control crystal growth of bioactive compounds. ${ }^{3}$

In order to produce such self-assembled nanowires, different strategies have been developed so far. Among them, most examples rely on the utilization of precipitation ${ }^{4}$ or gelation. ${ }^{5}$ Despite clear advantages (e.g. easy implementation), these methods, which rely on the propensity of the molecule to aggregate into well-defined objects, have in common to afford materials whose structuration strongly depends on the experimental conditions. For example, from the same derivative, completely different structures may be obtained by modifying the temperature, ${ }^{6}$ the substrate nature ${ }^{7}$ or the solvent of preparation ${ }^{8}$ or by introducing additives. ${ }^{9}$ Yet, the influence of these parameters on the structuration and thus on the physico-chemical properties remains rarely studied by materials chemists.

In the particular case of gelators, the shape of the corresponding materials has also clearly been neglected so far. In this regard,

\footnotetext{
${ }^{a}$ Université d'Angers, CNRS UMR 6200, Laboratoire MOLTECH-Anjou, 2 bd Lavoisier, 49045 Angers Cedex, France. E-mail: david.canevet@univ-angers.fr, narcis.avarvari@univ-angers.fr, marc.salle@univ-angers.fr

${ }^{b}$ Institut des Matériaux Jean Rouxel, 2 rue de la Houssiniére, BP32229,

44322 Nantes Cedex 3, France

$\dagger$ Electronic supplementary information (ESI) available: Additional optical and electronic micrographs, experimental conditions, NMR and mass spectra. See DOI: $10.1039 / \mathrm{c} 4 \mathrm{nj} 00681 \mathrm{j}$
}

some efforts have been made in order to get an aligned network of nanofibres. To do so, van Esch, Samorì and coworkers, for instance, described the utilization of a strong electric field, which was applied to a hot solution of a gelator that slowly cools down at the sol-gel transition temperature. ${ }^{10}$ The process allows for obtaining, at least, to a certain extent. In this context, Shinkai et al. also reported striking images of a gelator that spontaneously forms parallel nanowires of a tetrathiafulvalenebased derivative at very high concentrations $\left(\sim 100 \mathrm{mg} \mathrm{mL}^{-1}\right) .{ }^{11}$ Such an example is obviously of great importance but it is also clear that one can hardly foresee such a behavior from the chemical structure of the gelator.

On this ground, we got interested in the use of porous membranes ${ }^{12}$ and, in particular those made of alumina, since they have been reported to template the growth of monodimensional structures made up of very different kinds of materials, ${ }^{13}$ such as various covalent polymers, or even to produce organic-inorganic hybrid heterojunction nanowires or metal nanorods. ${ }^{14}$ This method relies on two key-steps: (i) filling the alumina membrane with the desired material and (ii) dissolving the alumina template in order to recover the shaped material (Fig. 1).

In the case of alumina membranes, track-etch techniques allow for generating pores, which display well-defined diameters, ${ }^{15}$ and thus afford shaped materials with a high monodispersity. Moreover, the diameter of the resulting objects is controllable by selecting an appropriate membrane $(20 \mathrm{~nm}<\varnothing<200 \mathrm{~nm})$.

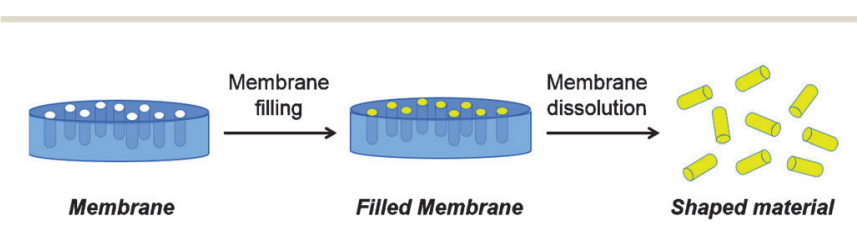

Fig. 1 Templated growth of nanocylindres with a membrane. 
Given these dimensions, the incorporation of nanofibers prepared from gels appears to be interesting especially as the pore itself could confine the fibers and constrain them into particular arrangements. Moreover, to the best of our knowledge there is no study available in the literature describing incorporation of organogels in such membranes. Therefore, from a fundamental point of view, this approach is of evident interest. A particularly important issue to address concerns the effect of the membrane on the structure of the grown material. This is the reason why we notably report herein our preliminary efforts to incorporate gelator-based nanofibers into membranes and dissolve the latter to promote an original way of obtaining well-defined structures. To do so, we selected two previously described gelators with simple structures, ${ }^{16}$ both of which contain two urea functions and a nonylene $\left(-\mathrm{C}_{9} \mathrm{H}_{18^{-}}\right)$spacer. Compound 1 displays benzyl moieties at its periphery while compound 2 is endowed with aliphatic dodecyl chains. Prior to considering their incorporation in membranes, the capability of these compounds to self-assemble in fibers appears to be strongly dependent on the solvent used, a parameter mentioned above and which has been thoroughly studied herein in the case of 1 and 2.

\section{Results and discussion}

\section{Synthesis}

Compounds 1 and 2 (Scheme 1) were synthesized according to the reported procedure, ${ }^{16}$ which involves the condensation of 1,9-diaminononane with two equivalents of benzyl isocyanate or dodecyl isocyanate, respectively. Their molecular structures have been properly confirmed by various techniques, including NMR, mass spectrometry, elemental analysis, infrared absorption spectroscopy and DSC, and the corresponding data are in perfect agreement with the literature.

\section{Organogelating properties and optical quality of the gels}

The organogelating properties of compounds $\mathbf{1}$ and $\mathbf{2}$ were studied in solvents (Table 1 ) with significantly different coordinates on a Teas diagram (Fig. S1, ESI $\dagger$ ). ${ }^{17}$ First, it appears that 2 forms gels in a wider range of solvents than $\mathbf{1}$. Indeed, among the eighteen tested solvents, compound $\mathbf{1}$ is able to generate gel phases in five of them, whereas compound 2 is able to gel fourteen solvents. No direct relationship seems to exist between gelation and the position of the solvent in the Teas diagram, which depends on the solvent ability to interact with a given solute through hydrogen bonding, dispersion or polar forces. On the other hand, a careful inspection of Table 1 allows us to

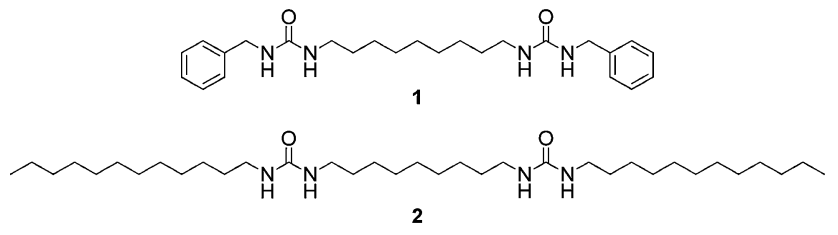

Scheme 1 Chemical structures of compounds 1 and $\mathbf{2}$. rationalize such a difference. First, one should note that a gel is systematically obtained with 2 when compound 1 does form a gel in a given solvent. Moreover, gelation by $\mathbf{1}$ is only observed with high boiling point solvents, which are as different as tetralin (1,2,3,4-tetrahydronaphthalene) (b.p. $\left.208{ }^{\circ} \mathrm{C}\right)$, chlorobenzene (b.p. $134{ }^{\circ} \mathrm{C}$ ), or octan-1-ol (b.p. $195{ }^{\circ} \mathrm{C}$ ). This suggests that high temperatures are required to dissociate the aggregates and to permit the growth of nanowires responsible for the gel formation by 1 . Moreover, consistent with the above observation, the melting point of compound 1 is higher (m.p. $(\mathbf{1})=198-200{ }^{\circ} \mathrm{C}$; m.p. $(2)=168-170{ }^{\circ} \mathrm{C}$ ), which corroborates the occurrence of stronger intermolecular interactions in the case of 1. Eventually, during the gelation tests performed using $\mathbf{1}$, we made an unusual observation regarding the temperature that had to be reached: with certain samples, heating until dissolution, i.e. until perfect eye transparency, was not sufficient to get gels after cooling down, and the heating had to be led further so as to form the gels. In light of the above-described observations, we conclude that compound 2 is a better gelator than 1. Nevertheless, one should have in mind that this assessment may not only result from the presence of long alkyl chains favouring intermolecular van der Waals interactions but may also result from the very poor solubility of bis(benzyl) derivative $\mathbf{1}$. In addition to the above qualitative comparisons, the measurement of the critical gelation concentration (CGC) provides useful quantitative data. These CGCs were determined following the inverted vial technique. ${ }^{19}$ Whatever the solvent and the gelator under study, most of the CGCs are comprised between 2 and $4 \mathrm{mg} \mathrm{mL}{ }^{-1}$, which corresponds to remarkably low values and which illustrates the very good propensity of such bis-urea systems to promote solvent gelation. However, there are three notable exceptions with CGC values comprised between 7 and $12 \mathrm{mg} \mathrm{mL}^{-1}$, which in common correspond to highly polar, non-aromatic solvents with a high dielectric constant and endowed with hydrogen bond donor or acceptor moieties, namely tert-butanol, $N, N$-DMF and octan-1-ol (Table 1). At this stage, it should be noted that this assessment seems to be remarkable, especially as these three solvents belong to a region of the Teas diagram, where no other solvent under consideration can be found (Fig. S1, ESI $\dagger$ ).

We are notably interested in organogels as propagation media for optical applications. In this context, a critical issue deals with the optical quality of the medium. Indeed, when aiming at quantifying optical phenomena, perfect transparency is desirable. If optical diffusion takes place, a part of the incident and outgoing photons are lost and not taken into account. As illustrated in Fig. 2 and 3, whatever the gelator under study is, the optical quality of the gels strongly depends on the utilized solvent. For instance, 1 forms an opaque gel in octan-1-ol and a transparent one in tetralin. There also exist intermediate situations, like the 1,2,4-trichlorobenzene-based organogel from $\mathbf{1}$, for which small white particles can be distinguished. Regarding compound 2, most gels are completely opaque (e.g. chloroform- or $p$-xylenebased organogels). However, there are a few notable exceptions: solvents that are both chlorinated and aromatic afford gels with a certain degree of transparency and, once again, the tetralin-based organogel displays the nicest optical quality. 
Table 1 Test for solubility and gel formation for compounds 1 and $\mathbf{2}$ - P stands for precipitate, I for insoluble at high temperatures, G for gel. In the case of the gels $\mathrm{G}(x), x$ corresponds to the $\mathrm{CGC}$ value in $\mathrm{mg} \mathrm{mL}^{-1}$, which is the minimum gelator amount required for gel formation at $20{ }^{\circ} \mathrm{C}$ per $\mathrm{mL}$ of the solvent

\begin{tabular}{|c|c|c|c|c|c|}
\hline Solvents & Compound 1 & Compound 2 & b.p. $\left({ }^{\circ} \mathrm{C}\right)$ & Dielectric constant $^{18}$ & Dipolar moment $(D)^{18}$ \\
\hline Tetrahydrofuran & I & G (2) & 66 & 7.52 & 1.75 \\
\hline tert-Butanol & $\mathrm{P}$ & $\mathrm{G}(7)$ & 82 & 12.4 & 1.7 \\
\hline Acetonitrile & I & I & 82 & 36.64 & 3.92 \\
\hline 1,4-Dioxane & I & G (3) & 101 & 2.219 & 0 \\
\hline$p$-Xylene & I & G (3) & 138 & 2.273 & 0 \\
\hline 1,1,2,2-Tetrachloroethane & $\mathrm{P}$ & G (4) & 146 & 8.5 & 1.32 \\
\hline$N, N$-Dimethylformamide & $\mathrm{P}$ & G (8) & 153 & 38.25 & 3.82 \\
\hline 1,2-Dichlorobenzene (oDCB) & G (2) & G (2) & 179 & 10.12 & 2.5 \\
\hline Dimethylsulfoxide & $\mathrm{P}$ & $\mathrm{P}$ & 189 & 47.24 & 3.96 \\
\hline Octan-1-ol & G (12) & G (10) & 195 & 11.3 & 1.72 \\
\hline Tetralin & G (3) & G (4) & 208 & 2.77 & 0 \\
\hline
\end{tabular}

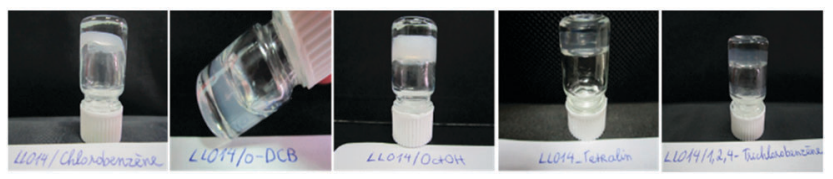

Fig. 2 Organogels obtained from 1 in the different solvents allowing for gelation.

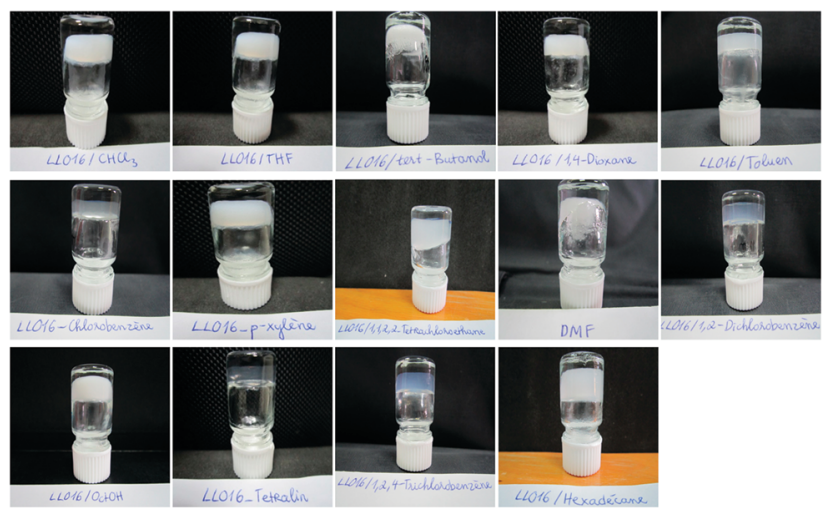

Fig. 3 Organogels obtained from 2 in the different solvents allowing for gelation.

\section{Influence of the solvent on the morphologies of the xerogels}

Given the differences observed between these organogels prepared from different solvents, optical microscopy appears to be a simple and relevant tool in order to get insight into the morphology of the fibre network responsible for gelation. At this stage, one should also note that previous reports have demonstrated the critical role of the solvent regarding the structures of the fibres and sometimes over their physical properties. ${ }^{8}$ Other important issues when aiming at imaging xerogels are the substrate nature ${ }^{7}$ and the casting process, parameters which are apparently rarely studied. In particular, the substrate may have a significant influence on the fibre arrangement. We used
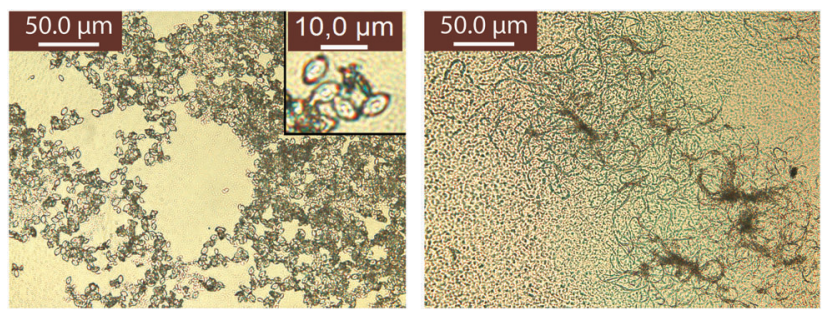

Fig. 4 Optical micrographs of 1-based xerogels prepared from chlorobenzene depending on the casting method. Left: deposition of a piece of gel (the inset in the top right corner corresponds to a magnification). Right: drop-casting (the corresponding gels were prepared at a concentration $C=1.25 \times(\mathrm{CGC})$.

two different deposition methods: (i) the first one limits the effect of the substrate and simply involves the deposition of a piece of gel on a glass slide; (ii) the second one (drop casting) consists in casting a hot drop of the gelator solution (at a concentration $C>\mathrm{CGC}$ ) on a glass slide, which cools down and forms a gel. From these two processes, evaporation of the solvent leads to samples which are observed under a microscope. The corresponding images are shown in Fig. 4 and Table S1 (ESI $\dagger$ ) for gelator $\mathbf{1}$ and Fig. 5 and Table S2 (ESI $\dagger$ ) for gelator 2. Regarding compound 1, a first assessment emanates from the comparison of the images depending on the casting mode. In each case, one can identify a network structural organization, which is characteristic of xerogels but the observed morphology is significantly, and sometimes, drastically different according to the deposition method. The most striking difference lies on the xerogels prepared from chlorobenzene (Fig. 4). When the material is drop-casted, a classic xerogel picture was obtained with the occurrence of fibres that have diameters close to $1 \mu \mathrm{m}$ and variable lengths reaching a few hundreds of micrometres. As commonly observed in xerogels, these organic wires are intertwined and randomly arranged. In contrast, when a piece of gel is deposited on the glass slide, a totally new situation arises. 


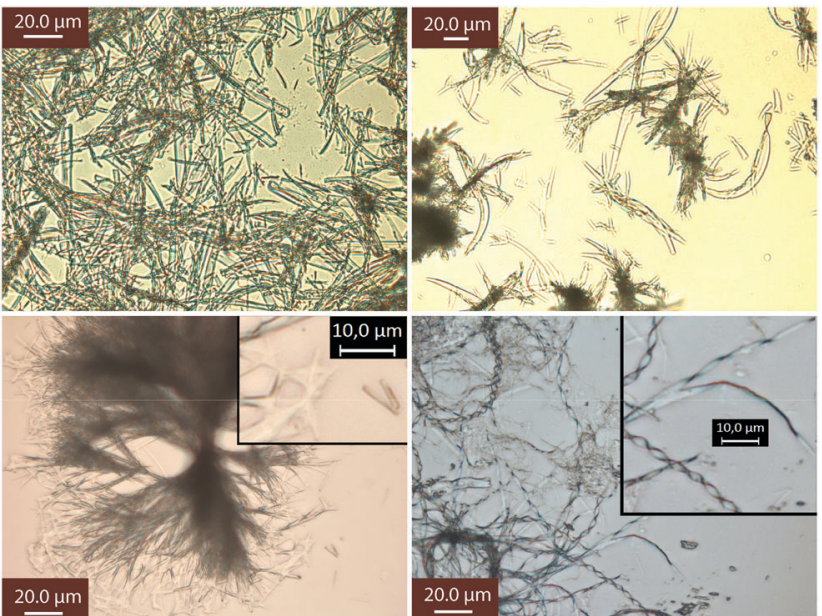

Fig. 5 Images of $\mathbf{2}$-based xerogels by optical microscopy depending on the solvent of preparation: $\mathrm{tBuOH}$ (top left), DMF (top right), $\mathrm{OctOH}$ (bottom left), hexadecane (bottom right) (the corresponding gels were prepared at a concentration $C=1.25 \times C G C$ and directly deposited on a glass slide - the insets in the top right corners correspond to a magnification).

Despite the networking tendency of the organic molecule, fibres are no longer observed. Instead, small interacting vesicles form the network. Interestingly, these particles seem to have very close morphologies and dimensions (see the inset in Fig. 4). Their shape is ovoid with sizes of $c a$. 6-7 $\mu \mathrm{m} \times 4 \mu \mathrm{m}$. Such a striking difference in the gel morphology for two samples of the same gel underlines the importance of the casting method, an issue which is not often taken into consideration. Though less pronounced, a similar behaviour is observable from the chemically close $o$-dichlorobenzene $(o \mathrm{DCB})$ solvent, for which fibres and microparticles coexist (see Table S1, ESI $\dagger$ ).

Concerning the other solvents (Table S1, ESI $\dagger$ ), the differences in gel morphologies $v s$. the casting method are less spectacular but still, significant. Eventually, it appears from these data that the films appear to be much more homogeneous and thinner when drop-casted, which makes them better candidates in the context of optical applications. As mentioned above, compound 2 is an excellent gelator, with a capability to gelate fourteen over eighteen tested solvents. The xerogels obtained after depositing a piece of organogel on a glass slide and subsequent evaporation of the solvent are presented in Fig. 5 and Table S2, ESI. $\dagger$ In most solvents, the networking tendency of compound 2 is easily observable: as expected, the compound classically weaves a web composed of intertwined self-assembled fibres with high aspect ratios (e.g. THF, 1,1,2,2-tetrachloroethane, oDCB, and tetralin). However, the cases of tert-butanol, DMF, octan-1-ol on the one hand, and hexadecane on the other hand, have drawn our attention. The first three solvents, which are in a peculiar area of the Teas diagram (Fig. S1, ESI $\dagger$ ), have been mentioned above regarding the related critical gelation concentrations since compound 2 displays significantly higher CGCs in these solvents. In the cases of DMF and tert-butanol, the observation of the micrographs presented in Fig. 5 shows that transparent crystallites are ubiquitous in these samples. Concerning octan1-ol, the situation is not so clear at first glance but a careful examination of the micrograph allows for distinguishing transparent microcrystals, which are ordered in bundles (see inset). Thus, on the basis of these assessments, we suggest that the corresponding higher CGCs may be linked to the tendency of compound 2 to crystallize in these solvents rather than to form self-assembled fibres with multiple intersections. As for hexadecane, it had already been identified as generating gels with compound 2 in a previous work. ${ }^{16}$ However, no optical micrograph was provided and the authors mentioned that they were not able to observe any junction zones between the selfassembled fibres. Probably because our sample preparation differed, we managed to observe a nice network composed of fibres, among which many helical ribbons, showing both leftand right-handed helicities, could be observed. These helices display various pitches and different diameters. For instance, a helix displaying a $6.5 \mu \mathrm{m}$ pitch presents a diameter of $2.8 \mu \mathrm{m}$ while another one with a $12.3 \mu \mathrm{m}$ pitch has a diameter of $1.7 \mu \mathrm{m}$ (see the inset). Regarding the samples prepared by dropcasting (Table S3, ESI $\dagger$ ), similar conclusions can be drawn since xerogels have significantly different structures depending on the solvent of preparation. For instance, the chlorobenzenebased xerogel displays an alveolate structure while the hexadecanebased one has a far more fibrillar microstructure. Altogether, these results show how critical is the deposition method and to which extent, associated with the solvent nature, these parameters can impact the shapes and the sizes of supramolecular aggregates.

\section{Proton nuclear magnetic resonance $\left({ }^{1} \mathrm{H}\right.$ NMR) and differential scanning calorimetry (DSC)}

Physical gels result from weak interactions which promote aggregation between gelator molecules. ${ }^{5 a}$ Therefore, providing energy to these systems usually allows for breaking of these aggregates and return to the solution state at higher temperatures (thermoreversibility). As a consequence, studying the aggregation process as a function of temperature appears to be a relevant strategy in order to get insight into the forces and thus the functional moieties involved in the gelation. ${ }^{20}$

To do so, two very different solvents were selected, namely dimethylsulfoxide (DMSO) and $o$-dichlorobenzene. The first reason justifying this choice came from the fact that both compounds 1 and 2 form gels in oDCB and not in dimethylsulfoxide, which is ascribed to the very different chemical structures and physicochemical properties of these solvents. For instance, DMSO is a strong hydrogen bond acceptor and is well-known for its ability to compete intermolecular hydrogen bonds, such as the ones existing between two urea functions. In contrast, $o$ DCB can only be considered as a weak hydrogen bond acceptor. Another reason for this choice is supported by the high boiling points of both solvents, which allow to led ${ }^{1} \mathrm{H}$ NMR studies on a very broad range of temperature, which is required to address the thermoreversibility issue of these systems.

The VT ${ }^{1} \mathrm{H}$ NMR experiment carried out from $293 \mathrm{~K}$ to $393 \mathrm{~K}$ shows that in DMSO-D 6 , compounds 1 and 2 display similar behaviours (Fig. 6). The NH signals (black and white circles for 

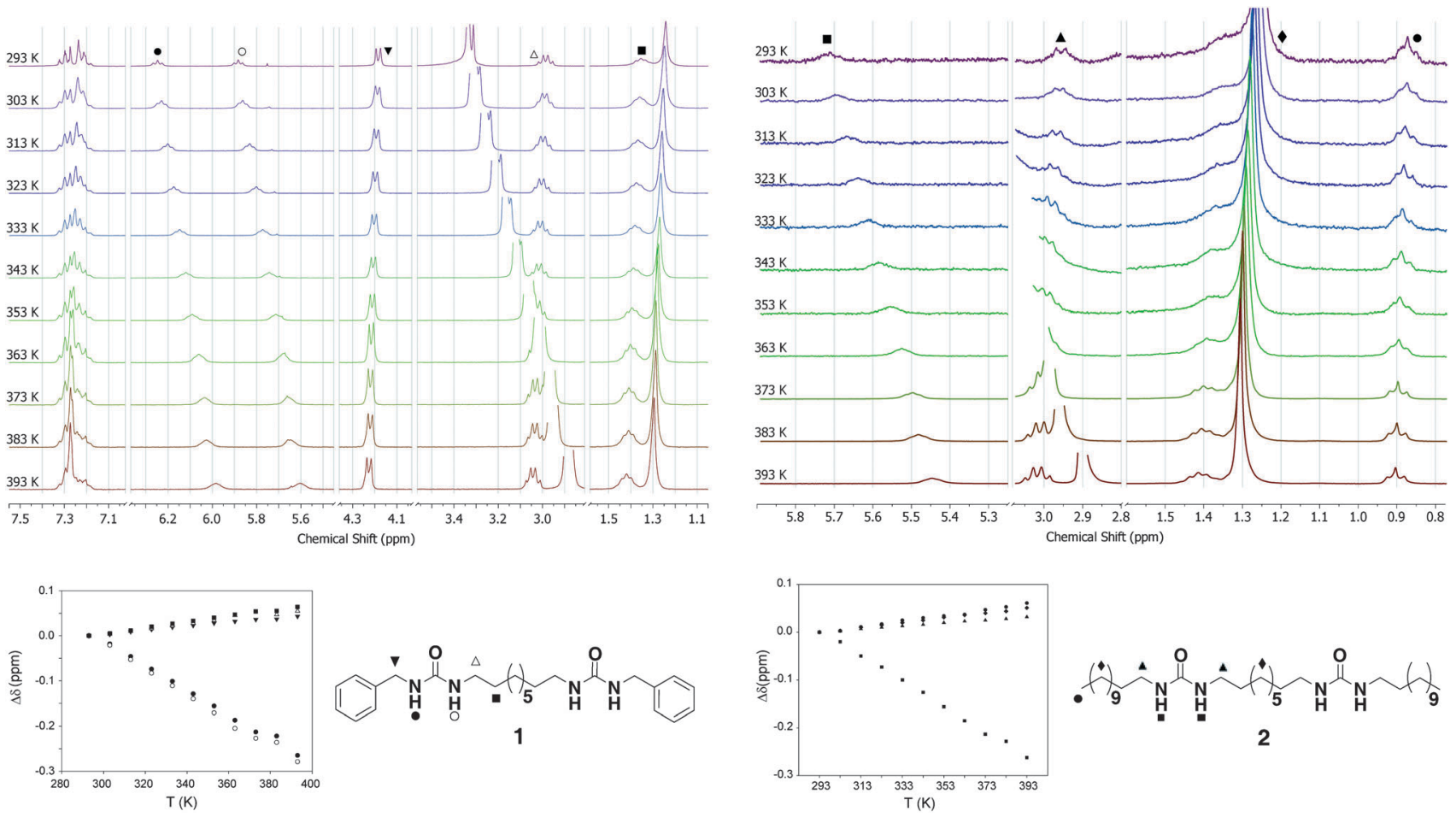

Fig. 6 Left column: $V T^{1} H$ NMR spectra of compound 1 in DMSO- ${ }_{6}\left(C=6.7 \mathrm{mg} \mathrm{mL}^{-1}-\right.$ water signal deleted for clarity) - bottom: variations in the chemical shifts of 1 as a function of temperature. Right column: VT ${ }^{1} \mathrm{H}$ NMR spectra of compound $\mathbf{2}$ in DMSO-D $\left(C=6.7 \mathrm{mg} \mathrm{mL}^{-1}-\mathrm{water}\right.$ signal deleted for clarity) - bottom: variations in the chemical shifts of $\mathbf{2}$ as a function of temperature.

1 and black squares for 2) are as expected, continuously upfieldshifted upon increasing the temperature. In other words, the lower the temperature, the stronger are the hydrogen bonds. The variation in chemical shift exceeds $\Delta \delta=0.25 \mathrm{ppm}$ in both cases, which is common for such systems. ${ }^{21}$ Interestingly, the protons from the aromatic rings in $\mathbf{1}$ do not undergo significant chemical shift variations. This is an important statement since previous studies have shown that aggregation through $\pi-\pi$ stacking is usually associated with a downfield shift of the corresponding proton signals. ${ }^{8 a}$ This suggests a weak contribution of these moieties in the aggregation process in 1 . This presumably results from the vicinity of the phenyl rings and the urea functions, which cannot act synergistically given the short and rigid methylene spacer. ${ }^{2 d}$ As for other signals, they are lowfield shifted upon heating the sample. This was expected since the long alkylene spacers do form lipophilic domains through van der Waals interactions at lower temperatures. In any case, since the chemical shift variations $\Delta \delta$ did not reach threshold values within the temperature range, it was unfortunately not possible to fit this evolution with a theoretical model to access the intermolecular binding constants.

In $o \mathrm{DCB}$, the situation proved to be more complicated because of solubility issues and therefore signals of lower resolution (Fig. 7). In order to be able to compare the experiments carried out in DMSO with those carried out in $o \mathrm{DCB}$, the concentrations of 1 and 2 were fixed at the same level $\left(6.7 \mathrm{mg} \mathrm{mL}^{-1}\right)$. Moreover, importantly, this concentration was chosen higher than the CGC value (case of $o \mathrm{DCB}$ ), in order to study the evolution of the gel state upon heating up the samples. The first difference came from the fact that the signal ascribed to the methylene bridge of the benzyl group in 1 undergoes an upfield shift from 4.36 to 4.29 ppm upon heating from $293 \mathrm{~K}$ to $393 \mathrm{~K}$, while a downfield shift was observed in DMSO. Therefore, two significantly different aggregation processes are clearly expected from these solvents. The $\mathrm{NH}$ signal progressively appears as a broad signal upon heating from $363 \mathrm{~K}$, and is clearly observed at $393 \mathrm{~K}(4.58 \mathrm{ppm})$. Therefore, its chemical shift decreases upon heating, which is consistent with the weakening of the hydrogen bonds at higher temperatures. The $\mathrm{NH}$ group in compound 2 seems to play a different role, since two distinctive temperature regimes are observed in oDCB. From $293 \mathrm{~K}$ to $353 \mathrm{~K}$, the signal is upfield-shifted from $4.18 \mathrm{ppm}$ to $4.01 \mathrm{ppm}$ and its integration decreases for the benefit of another broad signal, which appears in the 4.4-4.6 ppm region. This observation suggests the occurrence of an equilibrium between two hydrogen bonding modes. Given the presence of two urea functions in the molecule, a plausible explanation comes from interactions occurring in an intermolecular or an intramolecular fashion. ${ }^{22}$ Besides, certain molecules are well known for folding themselves upon heating since a temperature increase can lead to the desolvation of the solute (e.g. poly $\left.(N \text {-isopropyl-acrylamide })^{23}\right)$ which constitutes an argument in favour of an intramolecular hydrogen bonding at high temperatures in the case of compound 2.

Prior to determining the sol-gel transition temperatures of the gels, DSC measurements were performed on pure compounds. The results show that both bis-urea derivatives 1 and 2 are degraded upon melting (190 and $160{ }^{\circ} \mathrm{C}$ respectively - Fig. 8). 

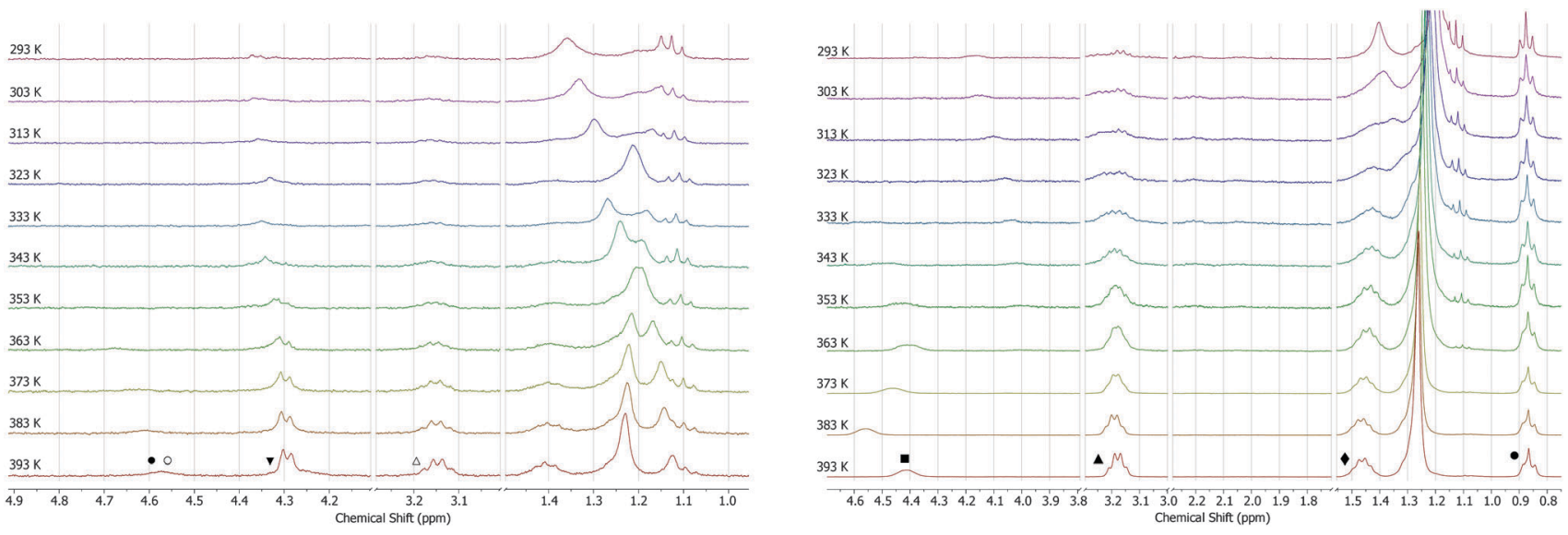

Fig. $7 \mathrm{VT}{ }^{1} \mathrm{H}$ NMR spectra of compounds 1 (left) and $\mathbf{2}$ (right) in oDCB-D 4 (signals of the aromatic part in $\mathbf{1}$ are hidden by the residual peaks of oDCB $\mathrm{C}=6.7 \mathrm{mg} \mathrm{mL}^{-1}$.

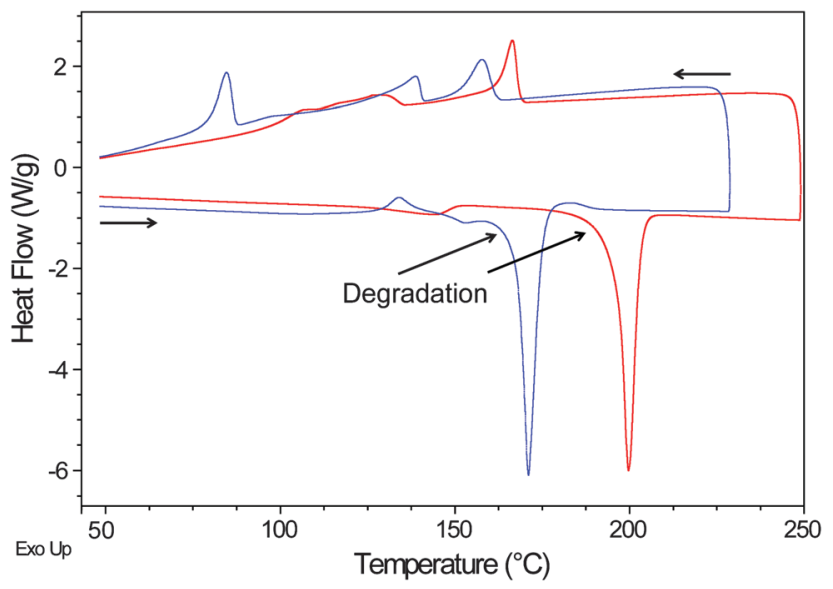

Fig. 8 DSC analyses of compounds 1 (red) and 2 (blue).

This degradation was confirmed by both ${ }^{1} \mathrm{H}$ NMR and mass spectrometry in the case of compound $\mathbf{1}$ after the sample was subjected to a $30{ }^{\circ} \mathrm{C}-220{ }^{\circ} \mathrm{C}-30{ }^{\circ} \mathrm{C}$ temperature cycle. The ${ }^{1} \mathrm{H}$ NMR analysis ( $3 \mathrm{mg} \mathrm{mL}{ }^{-1}-\mathrm{DMSO}^{-\mathrm{D}_{6}}$ ) also shows significant modifications: new signals appear at around the NH signals (5.5-6.25 ppm) and the spectral resolution is decreased (Fig. S4, ESI $\dagger$ ), as awaited from the very poor solubility of the samples after DSC analysis. With regard to the degradation reaction, various explanations can be considered since urea moieties, for instance, are well-known for self-reacting at high temperatures. ${ }^{24}$

DSC analyses were also conducted on gels prepared from various solvents. The temperature ranges have been chosen so as to maintain the samples at temperatures lower than the degradation ones of $\mathbf{1}$ and $\mathbf{2}$, as determined above. In most cases, the results were not reproducible but the experiments proved to be reliable for samples prepared from $o \mathrm{DCB}$ and TCB (1,2,4-trichlorobenzene) (see Table 2). With both solvents, compounds 1 and 2 form gels displaying close sol-gel transition temperatures as determined from DSC analysis. Obviously, the nature of the gelator has a critical role as shown by the large temperature difference observed for both gelators in the
Table 2 Transition temperatures obtained from DSC measurements; [gelator] $=2.4 \times 10^{-5} \mathrm{~mol} \mathrm{~L}^{-1}$

\begin{tabular}{lll}
\hline & Compound 1 & Compound 2 \\
\hline$o$ DCB & $137{ }^{\circ} \mathrm{C}$ & $104{ }^{\circ} \mathrm{C}$ \\
TCB & $135{ }^{\circ} \mathrm{C}$ & $111^{\circ} \mathrm{C}$
\end{tabular}

same solvent. These values range from $24{ }^{\circ} \mathrm{C}$ to $33{ }^{\circ} \mathrm{C}$ in TCB and $o \mathrm{DCB}$, respectively, even though the gelator concentration was the same $\left(2.4 \times 10^{-5} \mathrm{~mol} \mathrm{~L}^{-1}\right)$ in order to allow for a rigorous comparison.

\section{Efforts towards the structuration of self-assembled nanofibres using alumina membranes}

Over the last two decades, a tremendous number of photoactive and electroactive nanowires have been described. ${ }^{5 d}$ However, their implementation in devices remains a great challenge that the scientific community will have to tackle in the future. ${ }^{25}$ In this context, being able to align these wires in a controlled and predictable manner is of utmost importance. Regarding covalent polymers, the strategy consisting of shaping them by a template approach using alumina membranes has definitely demonstrated its efficiency, notably with conducting polymers. ${ }^{13}$ Since this approach has never been transferred to supramolecular polymers, we describe below our attempts, failures and successes in this direction.

Filling the alumina membrane with a covalent polymer. Before attempting to prepare nano-objects made up of xerogels, i.e. a supramolecular polymer, a control experiment was performed using a covalent azobenzene polymer, in order to check the viability of the method. For this purpose, we used a methacrylate polymer substituted with strongly absorbing azo dyes (namely 3-[4-[(E)-(4-[(2,6-dimethyl-pyrimidin-4-yl)amino]sulfonylphenyl)diazenyl]phenyl(methyl)amino]propyl units) ${ }^{26}$ According to the experimental procedure previously described, ${ }^{26}$ the alumina membrane was filled with a saturated solution of the polymer, dried and then dissolved in an aqueous solution of sodium hydroxide. After rinsing, dispersing the material in water and 


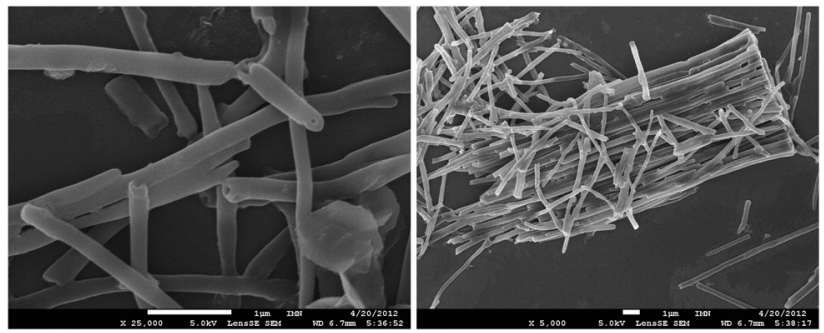

Fig. 9 SEM micrographs of the nanotubes obtained from the covalent methacrylate polymer.

dropping this suspension on a glass slide, the described method was successfully repeated, as illustrated by the SEM images shown in Fig. 9. Indeed, these nanotubes display the desired specifications and present a remarkable monodispersity regarding diameters.

Filling the alumina membrane with a supramolecular polymer. Of course, due to their inherent physico-chemical characteristics, a significantly different behaviour is expected for a supramolecular gel, compared to a solution of a covalent polymer. When considering the membrane filled with a physical gel, an intricate situation arises because of the pseudo-solid state of a gel. Indeed, its permeation through the alumina membrane is not as trivial as the one for a polymer solution (see above). At sufficiently high temperatures, physical gels melt to afford a solution but one should have in mind that this solution comes back to its initial state, the gel state, when the temperature decreases below the sol-gel transition temperature. Having these parameters in mind and looking for the most efficient filling method, various strategies were tested with gelator 2 - which displays the lowest solution to gel transition temperature - and different solvents: (i) a hot solution of the gelator was deposited onto a cold membrane ${ }^{27}$ (diameter: $25 \mathrm{~mm}$; thickness: $60 \mu \mathrm{m}$; diameter of the pores: $200 \mathrm{~nm}$ ); (ii) a hot solution was deposited onto a cold membrane under vacuum in order to facilitate the penetration of the solution through the pores; (iii) a hot solution was deposited onto a hot membrane in order to slow down the cooling process and maintain the solution state for a longer time; (iv) a hot solution was injected through a hot membrane under the pressure of a syringe; (v) a hot solution was injected in a cold membrane under the pressure of a syringe; (vi) the gel itself was directly injected onto a cold membrane using a syringe; (vii) the membrane was soaked in a hot solution of the gelator for a night; (viii) the membrane was placed over a drop of the hot solution, which was deposited on a hot glass slide; in this manner, the solution goes up through the pores thanks to capillary forces; (ix) three drops of the hot solution were successively deposited on a cold membrane in order to increase the amount of deposited material. We also considered the possibility to melt the pure compound on a heated membrane but DSC measurements demonstrate that the melting process was associated with a degradation of the gelator.

After drying, the two faces of the membranes as well as their slices were systematically observed by SEM microscopy in order to identify the most efficient strategy. The images obtained
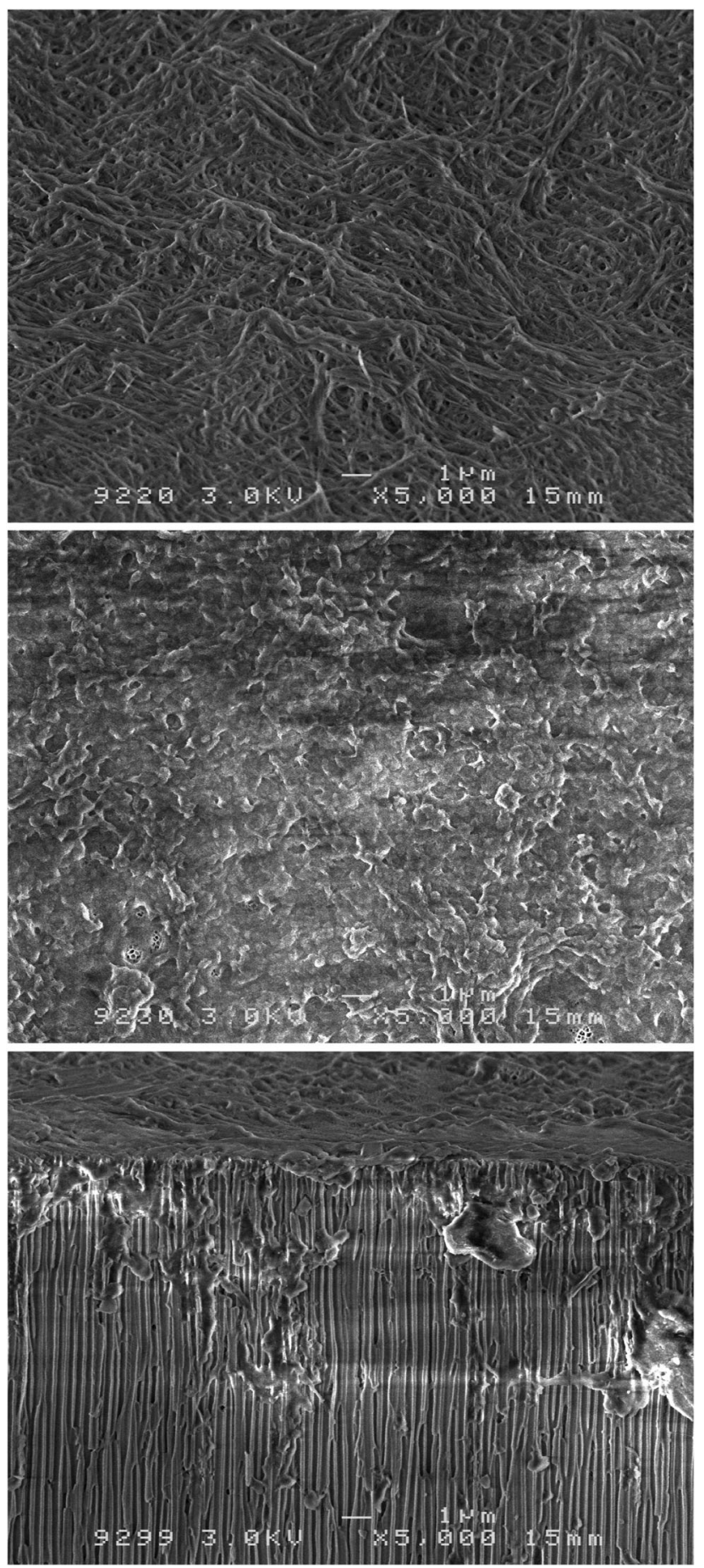

Fig. 10 SEM microscopy images of the alumina membrane in the presence of gelator $\mathbf{2}$ deposited according to strategy iv: upper face of the membrane (top), lower face (middle), slice (bottom).

from compound 2 and strategy iv are shown in Fig. 10, while the others are available in Table S4 (ESI $\dagger$ ). For all methods except viii, a large amount of xerogel was found on the upper face of the membrane, and importantly, the material was also found on the opposite face. Since the protocols we followed prevent the solution or the gel to bypass the membrane, the material necessarily crossed the membranes through the pores. However, 
when breaking the membranes, the SEM imaging of the slice could not evidence the presence of the xerogel in the pores. This may result from a poor contrast between the alumina membrane and the organic xerogel. Anyhow, the fact that no fibres could be observed indicates that the gelator may have formed a film on the pore walls. Given that SEM microscopy did not allow for detecting material in the membranes, Raman microspectroscopy was used to confirm the presence of the organic material within the pores. To do so, measurements were carried out (a) on a virgin membrane, (b) on a 2-based xerogel and (c) on a membrane slice prepared following the simplest method, namely i. In order to confirm the presence of a gelator within the pores, the alumina membrane was broken and studied using an Invia Renishaw spectrometer. The laser beam $(\lambda=632 \mathrm{~nm})$ was focused on the resulting membrane slice (thickness $=60 \mu \mathrm{m}$ ) with a diameter spot of $2 \mu \mathrm{m}$. Fig. 11 shows there is no Raman scattering coming from the virgin membrane. In contrast, the 2-based xerogel displays clear Raman bands at different wavenumbers (1447, 1293, 1130 and $1060 \mathrm{~cm}^{-1}$ ). Regarding the xerogel-membrane composite, one can observe that there are signals at different wavelengths. Some of these peaks can clearly be assigned to the xerogel materials $\left(\sim 1447\right.$ and $\left.1130 \mathrm{~cm}^{-1}\right)$ and others cannot. This assessment is critical since it definitely confirms the presence of xerogel material within the pores of the membrane but it also shows that new vibration modes $\left(e . g . \sim 810 \mathrm{~cm}^{-1}\right)$ exist when the xerogel is confined within the $200 \mathrm{~nm}$ wide pores.

Dissolution of the membranes. Using the membrane template-based deposition process opens up the possibility to isolate in fine monodimensional nano-objects (nanotubules and nanowires) of finite dimensions and that are monodisperse. To do so, isolation of the nano-objects is usually carried out by dissolution of the membrane subsequently to the filling step. This step needs therefore a prior removal of the material in excess, deposited on the upper and lower faces of the membrane. Accordingly, the membranes were wiped with a cotton soaked with the solvent used to prepare the gel. The dissolution of the alumina membrane is usually carried out by heating the filled membrane at $80{ }^{\circ} \mathrm{C}$ for

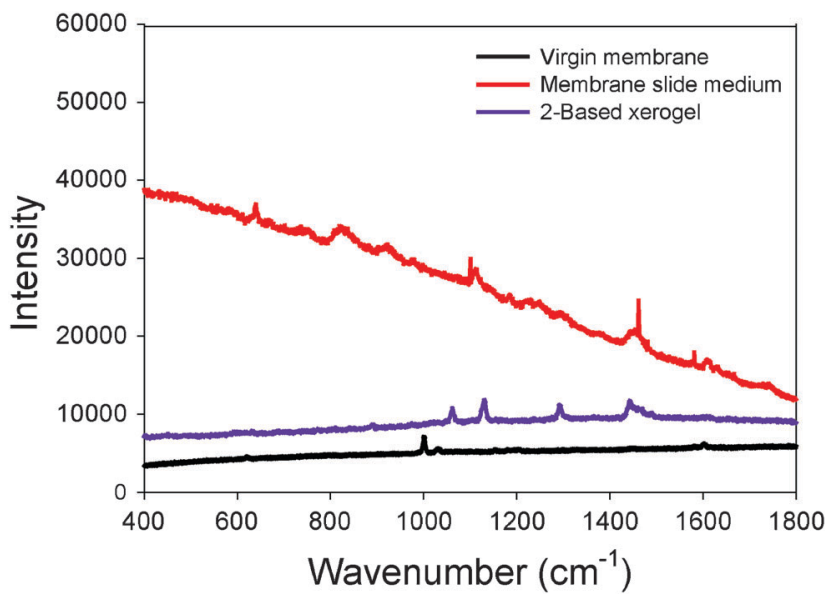

Fig. 11 Raman spectra recorded at $632 \mathrm{~nm}$ for the virgin membrane, the pure 2-based xerogel and the composite material modified membrane (pores containing 2-based xerogel). $T=300 \mathrm{~K}$; resolution $2 \mathrm{~cm}^{-1}$.

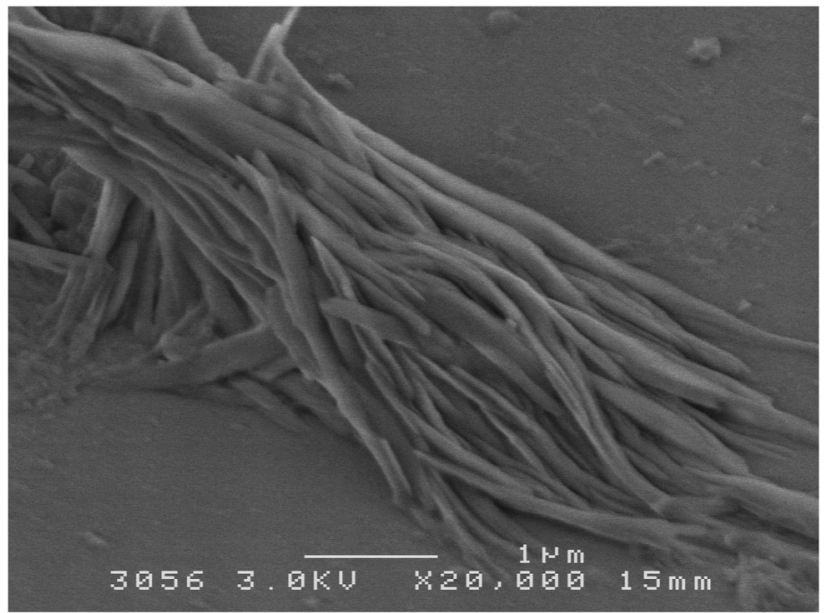

Fig. 12 SEM image of the 2-based material obtained after dissolution of the membrane.

several days. Given the structure of compound 2 and in particular the presence of urea functions, chemical evolution of the gelator under the basic conditions required for dissolving the alumina membrane cannot be excluded. A control experiment monitored by SEM microscopy and analysed by ${ }^{1} \mathrm{H}$ NMR spectroscopy showed that the 2-based xerogel remains intact upon heating for one week in a $5 \mathrm{M} \mathrm{NaOH}$ aqueous solution at $80^{\circ} \mathrm{C}$. In addition, this control experiment also demonstrates that self-assembled nanostructures based on 2, which are supported by a network of hydrogen bonds, are robust enough to resist to the highly competitive aqueous medium and are not disassembled upon standing in water (Fig. S5, ESI $\dagger$ ).

With this in mind, various composites 2@membrane were poured into a $5 \mathrm{M}$ sodium hydroxide solution until complete disappearance of the alumina. The suspension thus obtained was centrifuged and rinsed twice with distilled water. The material was eventually dispersed in water by sonication and a drop of the corresponding suspension was deposited on a clean glass slide. Among other morphologies, it is worth noting that we were able to observe nanowires homogeneous in size, of up to $10 \mu \mathrm{m}$ long and $c a .150 \mathrm{~nm}$ wide (Fig. 12), i.e. of dimensions in reasonable accordance with the specification of the alumina membranes used $(\varnothing=200 \mathrm{~nm}$, thickness $=40 \mu \mathrm{m}){ }^{28}$ These preliminary striking results constitute the first relevant indication of a template effect which is generated by a porous membrane over a xerogel-based material.

\section{Conclusions}

In summary, we have thoroughly explored the organogelating properties of two simple urea-based organogelators and highlighted a strong solvent-microstructure dependency. On this ground, we endeavoured to incorporate supramolecular polymers, i.e. physical gels, within alumina membranes to shape these materials. Preliminary results from these non-functionalized organogel models are particularly promising since for the first time, this template process could afford a collection of fibres 
homogeneous in size. A limitation of this approach lies on the very small amount of organic material which is deposited onto the walls of the pores within the membrane. From this auspicious preliminary study performed using model organogels, we are currently extending this new approach to gelators of higher critical gelation concentration in order to increase the amount of deposited material. In addition, the high potential of this original process in terms of material nanostructuration encourages us to further study this approach with organic functional gelators, in particular, endowed with specific optical properties. Work is in progress in this direction.

\section{Experimental}

\section{Materials and methods}

The starting materials were purchased commercially and were used without further purification. Compounds 1 and 2 were synthesized according to the literature. ${ }^{16}{ }^{1} \mathrm{H}$ and ${ }^{13} \mathrm{C}$ NMR spectra were recorded using a partially deuterated solvent as an internal reference on a BRUKER Advance DRX 300 spectrometer. Mass spectra were recorded on a Bruker Biflex III MALDI-TOF spectrometer. Alumina membranes (Anodisc) were obtained from Whatman and displayed the following characteristics: diameter: $25 \mathrm{~mm}$; thickness: $60 \mu \mathrm{m}$; diameter of the pores: $200 \mathrm{~nm}$. SEM images were acquired by scanning electron microscopy (SEM) JEOL JSM 6301F operating at tensions comprised between 0.5 and $30 \mathrm{kV}$. Optical micrographs were recorded on a LEICA DM2500P microscope by depositing the sample on glass slides.

\section{Procedure for the dissolution of the membrane}

To dissolve the alumina membranes, the latter were immersed in an aqueous $5 \mathrm{M} \mathrm{NaOH}$ solution at room temperature until complete disappearance of the alumina. The mixture was centrifuged, and the solid part was rinsed three times with distilled water. The material was eventually dispersed in a few milliliters of distilled water and deposited on a clean glass slide.

\section{Acknowledgements}

Authors acknowledge the Région des Pays de la Loire for a PhD grant (T.-L. L.) and, more globally, for funding the PHOTOGEL project. They are also grateful to the SCIAM (University of Angers) for microscopy facilities. Authors would also like to thank Pr Jean-Luc Duvail (Univ. Nantes) for his relevant and constructive remarks.

\section{Notes and references}

1 (a) Self-Assembled Nanomaterials I, ed. T. Shimizu, Berlin, Heidelberg, 2008; (b) A. P. H. J. Schenning and E. W. Meijer, Chem. Commun., 2005, 3245-3258; (c) A. R. Hirst, B. Escuder, J. F. Miravet and D. K. Smith, Angew. Chem., Int. Ed., 2008, 47, 8002-8018.
2 (a) F. Fages, J. A. Wytko and J. Weiss, C. R. Chim., 2008, 11, 1241-1253; (b) F. S. Kim, G. Ren and S. A. Jenekhe, Chem. Mater., 2011, 23, 682-732; (c) D. González-Rodríguez and A. P. H. J. Schenning, Chem. Mater., 2010, 23, 310-325; (d) T. Kitamura, S. Nakaso, N. Mizoshita, Y. Tochigi, T. Shimomura, M. Moriyama, K. Ito and T. Kato, J. Am. Chem. Soc., 2005, 127, 14769-14775; (e) J. Puigmartí-Luis, V. Laukhin, Á. Pérez Del Pino, J. Vidal-Gancedo, C. Rovira, E. Laukhina and D. B. Amabilino, Angew. Chem., Int. Ed., 2007, 46, 238-241; $(f)$ I. Dánilá, F. Riobé, J. Puigmartí-Luis, À. Pérez Del Pino, J. D. Wallis, D. B. Amabilino and N. Avarvari, J. Mater. Chem., 2009, 19, 4495-4504; $(g)$ J. Puigmartí-Luis, E. E. Laukhina, V. N. Laukhin, Á. Pérez Del Pino, N. Mestres, J. Vidal-Gancedo, C. Rovira and D. B. Amabilino, Adv. Funct. Mater., 2009, 19, 934-941; (h) D. Canevet, A. Pérez del Pino, D. B. Amabilino and M. Sallé, Nanoscale, 2011, 3, 2898-2902; ( $i$ ) I. Danila, F. Riobé, F. Piron, J. Puigmartí-Luis, J. D. Wallis, M. Linares, H. Ågren, D. Beljonne, D. B. Amabilino and N. Avarvari, J. Am. Chem. Soc., 2011, 133, 8344-8353; (j) I. Danila, F. Pop, C. Escudero, L. N. Feldborg, J. Puigmarti-Luis, F. Riobé, N. Avarvari and D. B. Amabilino, Chem. Commun., 2012, 48, 4552-4554; (k) K. K. Kartha, R. D. Mukhopadhyay and A. Ajayaghosh, Chimia Int. J. Chem., 2013, 67, 51-63.

3 (a) J. A. Foster, M.-O. Piepenbrock, M. G. O. Lloyd, N. Clarke, J. A. K. Howard and J. W. Steed, Nat. Chem., 2010, 2, 1037-1043; (b) F. Aparicio, E. Matesanz and L. Sanchez, Chem. Commun., 2012, 48, 5757-5759.

4 R. Van Hameren, A. M. Van Buul, M. A. Castriciano, V. Villari, N. Micall, P. Schön, S. Speller, L. M. Scolaro, A. E. Rowan, J. A. A. W. Elemans and R. J. M. Nolte, Nano Lett., 2008, 8, 253-259.

5 (a) P. Terech and R. G. Weiss, Chem. Rev., 1997, 97, 3133-3159; (b) L. A. Estroff and A. D. Hamilton, Chem. Rev., 2004, 104, 1201-1217; (c) N. M. Sangeetha and U. Maitra, Chem. Soc. Rev., 2005, 34, 821-836; (d) S. S. Babu, V. K. Praveen and A. Ajayaghosh, Chem. Rev., 2014, 114, 1973-2129.

6 J. F. Toro-Vazquez, J. Morales-Rueda, A. Torres-Martínez, M. A. Charó-Alonso, V. A. Mallia and R. G. Weiss, Langmuir, 2013, 29, 7642-7654.

7 S. Prasanthkumar, A. Gopal and A. Ajayaghosh, J. Am. Chem. Soc., 2010, 132, 13206-13207.

8 (a) D. Canevet, A. Pérez Del Pino, D. B. Amabilino and M. Sallé, J. Mater. Chem., 2011, 21, 1428-1437; (b) D. Dasgupta, S. Srinivasan, C. Rochas, A. Ajayaghosh and J.-M. Guenet, Soft Matter, 2011, 7, 9311-9315; (c) P. A. Korevaar, C. Schaefer, T. F. A. de Greef and E. W. Meijer, J. Am. Chem. Soc., 2012, 134, 13482-13491.

9 (a) D. Dasgupta, S. Srinivasan, C. Rochas, A. Ajayaghosh and J. M. Guenet, Langmuir, 2009, 25, 8593-8598; (b) S. Srinivasan, S. S. Babu, V. K. Praveen and A. Ajayaghosh, Angew. Chem., Int. Ed., 2008, 47, 5746-5749.

10 L. Sardone, V. Palermo, E. Devaux, D. Credgington, M. De Laos, G. Marletta, F. Cacialli, J. van Esch and P. Samorì, Adv. Mater., 2006, 18, 1276-1280. 
11 T. Kitahara, M. Shirakawa, S. I. Kawano, U. Beginn, N. Fujita and S. Shinkai, J. Am. Chem. Soc., 2005, 127, 14980-14981.

12 (a) C. R. Martin, Science, 1994, 266, 1961-1966; (b) C. R. Martin, Chem. Mater., 1996, 8, 1739-1746.

13 F. S. Kim, G. Ren and S. A. Jenekhe, Chem. Mater., 2010, 23, 682-732.

14 T. R. Kline, M. Tian, J. Wang, A. Sen, M. W. H. Chan and T. E. Mallouk, Inorg. Chem., 2006, 45, 7555-7565.

15 A. Huczko, Appl. Phys. A: Mater. Sci. Process., 2000, 70, 365-376.

16 J. van Esch, S. De Feyter, R. M. Kellogg, F. De Schryver and B. L. Feringa, Chem. - Eur. J., 1997, 3, 1238-1243.

17 (a) C. M. Hansen, Hansen Solubility Parameters: A User's Handbook, CRC Press, Boca Raton, FL, 2007; (b) M. Raynal and L. Bouteiller, Chem. Commun., 2011, 47, 8271-8273.

18 J. A. Dean, Lange's Handbook of Chemistry, 15 edn, 1999.

19 J. Brinksma, B. L. Feringa, R. M. Kellogg, R. Vreeker and J. van Esch, Langmuir, 2000, 16, 9249-9255.

20 G. Yu, X. Yan, C. Han and F. Huang, Chem. Soc. Rev., 2013, 42, 6697-6722.
21 (a) J. L. López, E. M. Pérez, P. M. Viruela, R. Viruela, E. Ortí and N. Martín, Org. Lett., 2009, 11, 4524-4527; (b) K. Yabuuchi, E. Marfo-Owusu and T. Kato, Org. Biomol. Chem., 2003, 1, 3464-3469.

22 (a) L. Bouteiller, O. Colombani, F. Lortie and P. Terech, J. Am. Chem. Soc., 2005, 127, 8893-8898; (b) L. Fischer and G. Guichard, Org. Biomol. Chem., 2010, 8, 3101-3117.

23 R. Liu, M. Fraylich and B. Saunders, Colloid Polym. Sci., 2009, 287, 627-643.

24 E. Delebecq, J.-P. Pascault, B. Boutevin and F. Ganachaud, Chem. Rev., 2012, 113, 80-118.

25 S. S. Babu, S. Prasanthkumar and A. Ajayaghosh, Angew. Chem., Int. Ed., 2012, 51, 1766-1776.

26 R. Barillé, P. Tajalli, S. Zielinska, E. Ortyl, S. Kucharski and J. M. Nunzi, Appl. Phys. Lett., 2009, 95, 053102.

27 "Cold membrane" means a membrane which is kept at room temperature.

28 Differences may result from sonication, which is necessary to disperse the material. 